\title{
Fully-Diverse Multiple-Antenna Signal Constellations and Fixed-Point-Free Lie Groups
}

\author{
Babak Hassibi \\ Department of Electrical \\ Engineering \\ California Institute of Technology \\ Pasadena, CA 91125 USA \\ e-mail: hassibi@caltech.edu
}

\author{
Mohammad Khorrami \\ Institute for Studies in Theoretical \\ Physics and Mathematics \\ P.O. Box 5531 \\ Tehran, 19395 IRAN \\ e-mail: mamwad@iasbs.ac.ir
}

\begin{abstract}
A group of unitary matrices is called fixed-point-free (fpf) if all non-identity elements of the group have no eigenvalues at unity. Such groups are useful in multiple-antenna communications, especially in multiple-antenna differential modulation, since they constitute a fully-diverse constellation. In [1] all finite fpf groups have been classified. In this note we consider infinite groups and, in particular, their most interesting case, Lie groups. Two such fpf Lie groups are currently widely used in communications: the group of unit modulus scalars, from which various phase modulation schemes, such as QPSK, are derived, and the $2 \times 2$ orthogonal designs of Alamouti, on which many two-transmit-antenna schemes are based. In Lie-group-theoretic jargon these are referred to as $U(1)$ and $S U(2)$. A natural question is whether there exist other fpf Lie groups. We answer this question in the negative: $U(1)$ and $S U(2)$ are all there are.
\end{abstract}

\section{SUMMARY}

One method to communicate with multiple antennas is to differentially encode the transmitted data using unitary matrices at the transmitter, and to differentially decode without knowing the channel coefficients at the receiver. Since channel knowledge is not required at the receiver, such schemes are ideal when channel tracking is undesirable and/or infeasibleeither because of rapid changes in the channel characteristics or because of limited system resources.

The probability of error of mistaking the transmitted unitary matrix $V_{\ell}$ with another unitary matrix in the signal constellation, say $V_{\ell^{\prime}}$, at high SNR is dominantly dependant on $\left|\operatorname{det}\left(V_{\ell}-V_{\ell^{\prime}}\right)\right|$. Therefore the problem of constellation design reduces to the following: given $M$ transmit antennas and transmit rate $R$, find a set $\mathcal{V}=\left\{V_{1}, \ldots, V_{L}\right\}$ of $L=2^{M R}$, $M \times M$ unitary matrices, such that the minimum of the absolute value of the determinant of their pairwise differences is as large as possible. Any constellation $\mathcal{V}$ with the property that the determinants of the pairwise differences are all nonzero, is called fully diverse.

In its full generality, exact solutions to the design problem appear to be intractable. To break the logjam, [1] investigated the case where $\mathcal{V}$ forms a group under matrix multiplication. In this case, the condition that the constellation be fully-diverse reduces to the condition that the group be fixed-point-free.

Definition 1 (Fixed-Point-Free Group) $A$ group $\mathcal{G}$ is called fixed-point-free (fpf) if it has a representation as unitary matrices with the property that the representation of each non-unit element of the group has no eigenvalue at unity.
[1] classifies all finite fpf groups, some of which result in constellations with excellent performance. However, the best constellations are not obtained for very high rates or for a large number of antennas. This brings up the question of whether there exist any infinite fpf groups? It turns out that there do (and they are widely-used in communications), though they were never thought of as fpf groups.

The first is $U(1)$, the group of unit-modulus complex scalars: $e^{j \omega}, \omega \in[0,2 \pi[$. It is used in PM, FM, single-antenna differential modulation, etc., and its optimal constellations are the well known $r$-PSK signals. The second is $S U(2)$, the group of $2 \times 2$ unit-determinant unitary matrices. It corresponds to Alamout's well known orthogonal design for two-transmitantenna systems, which has also been suggested for multiantenna differential modulation.

But are there any other infinite fpf groups? Using Levi's decomposition of all Lie algebras, along with Cartan's classification of all semi-simple Lie algebras, we have been able to answer this question for Lie groups.

Theorem 1 (All Fixed-Point-Free Lie Groups) The only fpf Lie groups are $U(1)$ and $S U(2)$. Their only fpf irreducible representations are 1-and 2-dimensional, respectively.

This is a negative result: the only fpf Lie groups are the ones currently employed in practice. Therefore our investigation has not led to any new group or new constellation. However, it suggests the following venue for further work and investigation: relax the fpf condition and classify all Lie groups whose non-unit elements have no more than $k>0$ unit eigenvalues. ( $k=0$ corresponds to fpf groups.) Since in designing a constellation of finite size one needs to sample the Lie group's underlying manifold, the rationale is that if $k$ is small then there is a good chance that the resulting sampled constellation will be fully diverse. As a first step in this direction, we have obtained the following result.

Theorem 2 (Lie Groups with Unitary Representations) A Lie group has a representation as unitary matrices if, and only if, it is a compact semi-simple group or the direct sum of $U(1)$ and a compact semi-simple group.

\section{References}

[1] B. Hassibi, A. Shokrollahi, B. Hochwald, and W. Sweldens, "Representation theory for high-rate multiple-antenna code design," to appear in IEEE Trans. Info. Theory, 2001. 